\title{
Reply to the Comment by G. A. Maul and M. Sidran
}

\section{ANDING and R. KAUTH}

Institute of Science and Technology, University of Michigan, Ann Arbor, Michigan

We agree with the two major points made by Maul and Sidran. First, the water vapor transmission model used determines the spectral band pair which yields the best estimates of the sea surface temperature. Secondly, the spectral bands given in our paper of 1970 will not provide the necessary information to obtain improved estimates of sea temperature. This is not to imply that the two band concept is invalid, only that we have since found that the water vapor transmission model used in our previous analysis is not representative of reality. We have since altered the transmission model and obtained a new result which is presented herein.

Bignell (1970) published a comprehensive dissertation on the absorption by water vapor throughout the spectral region from 8 to $20 \mu \mathrm{m}$. Bignell demonstrated that three types of absorption occur in the window region: (a) that due to local lines, (b) a continuum caused by the wings of water vapor lines within the $6.3 \mu \mathrm{m}$ band and the rotational water band which are pressure broadened by foreign gases, and (c) a continuum caused by water vapor lines which are selfbroadened. The continuum absorption coefficient at temperature $T$, total pressure $P$, and water vapor partial pressure $p$, is given by

$$
k(T, P, p)=k_{1} P+k_{2} p,
$$

where

$k_{1}$ is the absorption coefficient for foreign broadening at unit total pressure;

$k_{2}$ is the absorption coefficient for self-broadening at unit water vapor partial pressure.

Values of $k_{1}$ and $k_{2}$ determined by Bignell (1970) are given in Fig. 1. Bignell's results clearly indicate that the water vapor model used in our previous analysis did not include the effects of absorption caused by self-broadening, which is

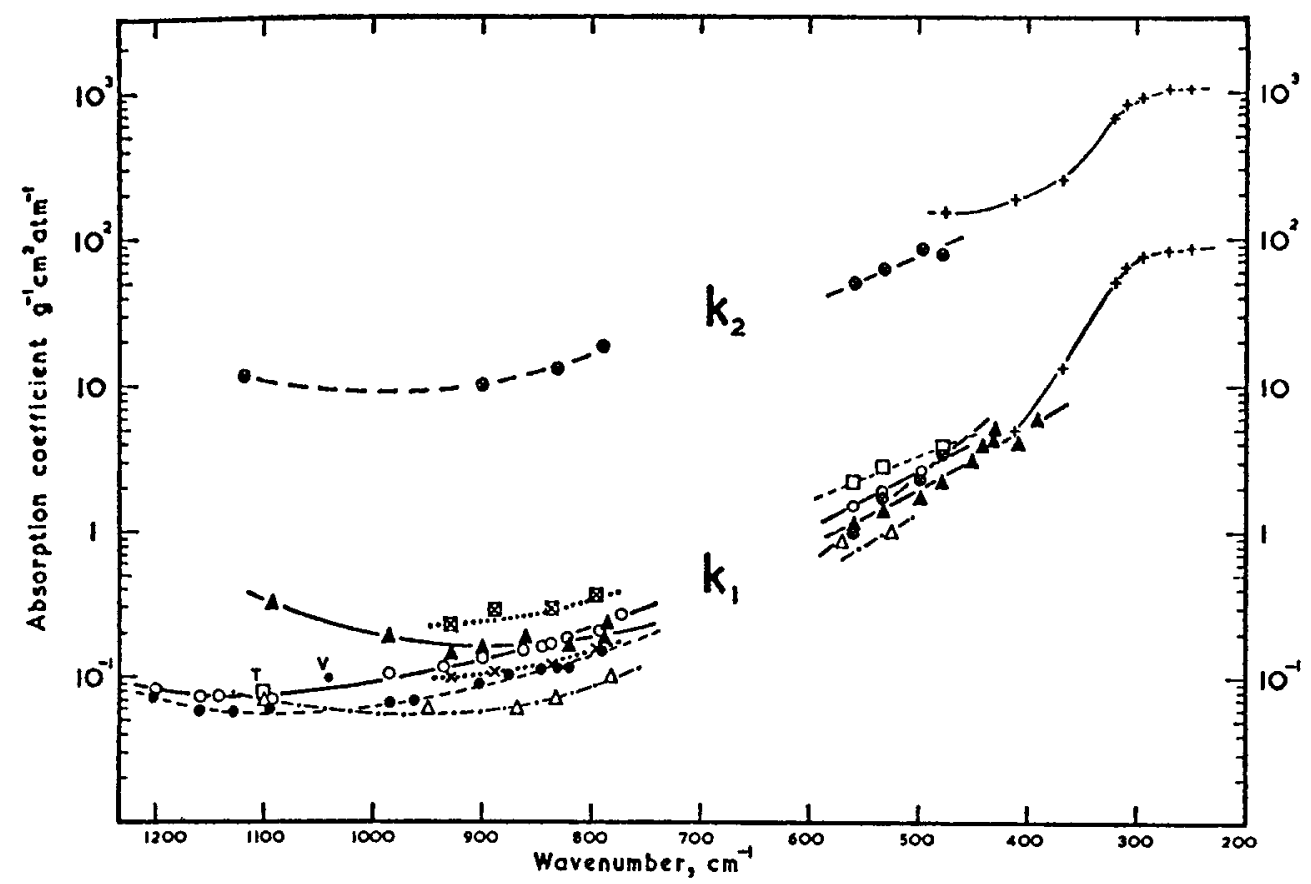

Fig. 1. Mass Absorption Coefficients (See Eq. 1) For The Foreign-Broadened $\left(k_{1}\right)$ and Self-Broadened $\left(k_{2}\right)$ Continuum. [Bignell] 
significant for the atmospheric paths considered in the sea temperature study. This is also true of the model used by Maul and Sidran, but to a lesser extent. The latter model was based upon the data of Roach and Goody (1958), which included water vapor partial pressures at sea level as high as $15 \mathrm{mb}$. Therefore, some selfbroadened-type absorption was reflected in the Roach and Goody data, but not at sufficient levels to define the value of $k_{2}$ accurately. It appears, therefore, that since neither our previous model, or the model used by Maul and Sidran, accurately represented the effects of self-broadening, neither result is acceptable.

In an effort to obtain a water vapor transmission model that provided for a better representation of reality, we have now used the data of Bignell for values of $k_{1}$ and $k_{2}$ in conjunction with the previously used values of Altshuler (1961) for local line absorption and have generated a new water vapor transmission model. Through the use of this new model and the statistical procedures described in our previous paper, a new two-channel pair was defined. The respective spectral bands are centered at 8.95 and $11.9 \mu \mathrm{m}$ and have band widths of 0.5 and $1.0 \mu \mathrm{m}$, respectively. For each of these spectral bands, inband radiance calculations for the five model atmospheres defined in our previous paper, for each of five sea surface temperatures and three zenith angles were performed ( 75 cases in all).

Although there is more spread in the points than previously shown, the data are still reasonably well represented by straight nearly parallel lines. The standard error in the estimate of the sea surface temperature is now $1.59^{\circ} \mathrm{K}$ rather than $0.15^{\circ} \mathrm{K}$ shown previously. As shown in Fig. 2, this increased error arises primarily from two sources: (a) observations at large zenith angles without using zenith angle as a variable in the estimation rule, and, (b) large differences between the sea surface temperature and the temperature of the lower atmosphere. In Fig. 2, the $\times$ 's are points for which the zenith angles are

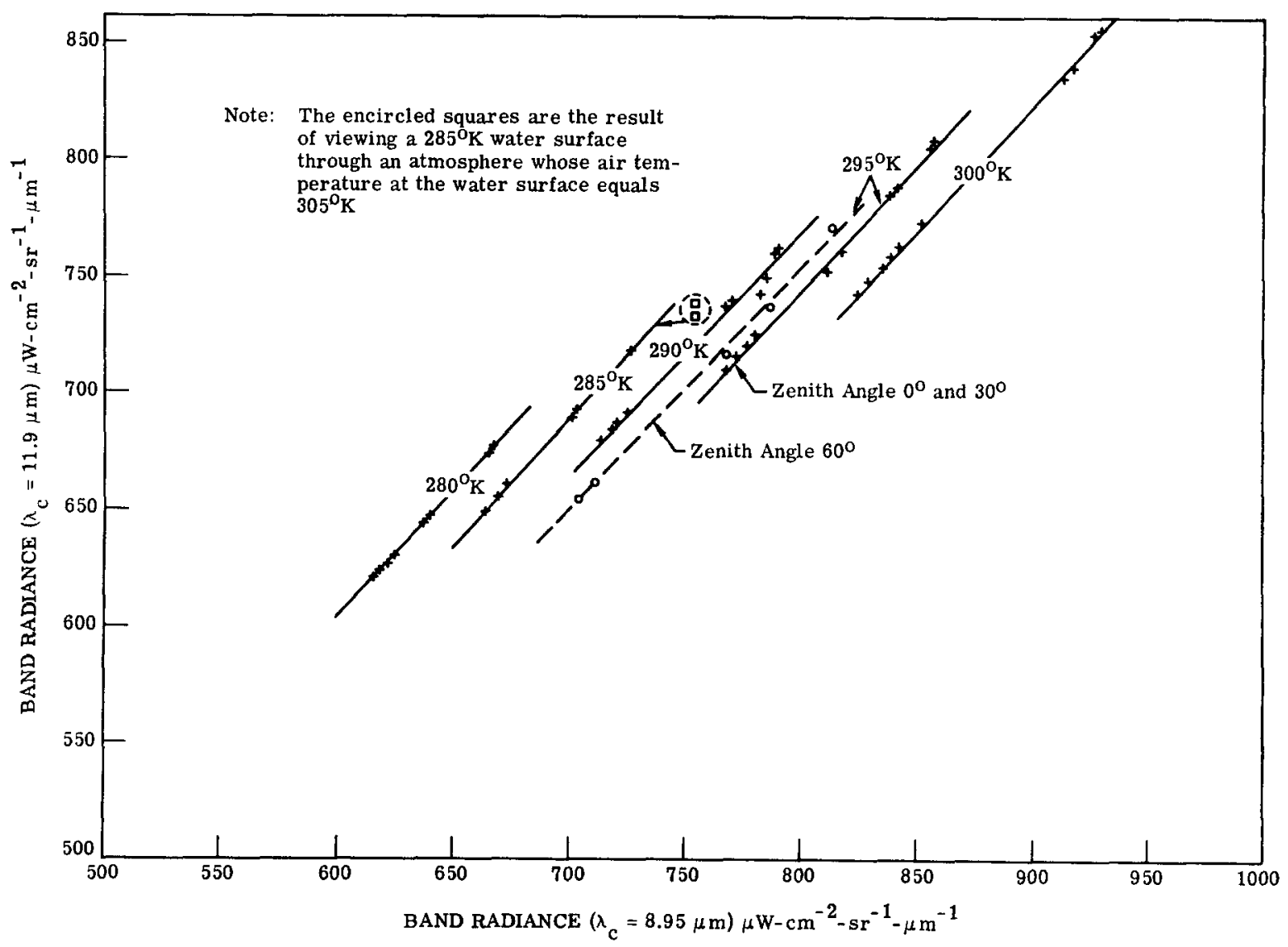

FIG. 2. Spectral Radiance in Band 1 vs. that in Band 2 as a Function of Atmospheric State. Parameters $=$ zenith angle at Surface and Sea Surface Temperature. 
0 and $30^{\circ}$ and for which the difference between the surface temperature and atmosphere temperature at the surface is less than $10^{\circ} \mathrm{K}$. If only this set of data points is included in the calculation, the standard error in the estimate of sea surface temperature is $0.45^{\circ} \mathrm{K}$.

The circles in Fig. 2 are points for a zenith angle of $60^{\circ}$. They are shown for only one surface temperature, $295^{\circ} \mathrm{K}$, to avoid cluttering the figure. Note that these points are well represented by a straight line, parallel to the others, but displaced. It is easy to see that if these points are accounted for by using a third observable, namely the zenith angle, in the estimation procedure the standard error can be reduced to near that for the 0 and $30^{\circ}$ cases alone.

The squares in Fig. 2 are points for which the surface temperature is greater than $10^{\circ} \mathrm{K}$ different from the temperature of the atmosphere at the surface. Again, only one case, surface temperature equal to $285^{\circ} \mathrm{K}$, is called out in the figure. In the calculation of standard error using all data these points were given equal weight with all others, while in the real world these points are quite unlikely to occur.

It is encouraging to note that even though the transmission models used by the authors are significantly different from each other (and also significantly different from that used by Maul and Sidran), the analysis performed on the data resulting from each model yielded two spectral bands (different for each case), which provided the necessary information to obtain a significant improvement in the estimate of the sea surface temperature over that obtainable from a single band measurement. It appears, therefore, that regardless of the spectral distribution of the absorption and emission of atmospheric water vapor in the spectral region of concern, a pair of spectral bands can be found which can achieve improved estimates of the sea surface temperature over that obtainable with a single band instrument. The problem is to determine the spectral bands which will yield the optimum results. If this is achieved through the use of simulated data, the credibility of the ir system depends upon the degree to which the water vapor transmission model represents reality. It would appear that the latest result is more credible than previous ones since it is based upon a water-vapor model which is in agreement with the latest accepted absorption data on self-broadened water vapor. The model does, however, lack verification and the results should not, therefore, be accepted as final.

The first spaceborne test of a two-channel procedure for estimating sea surface temperature will conceivably occur during the Skylab missions. The authors propose to estimate sea surface temperature for various two-channel pairs from data acquired by the S-191 interferometer spectrometer for sea surface test sites for which the sea surface temperature and atmospheric meteorology are accurately known. The test area (approximately one mile square) will be acquired 45 degrees forward of the spacecraft and tracked until the test area is 15 degrees aft. Approximately 60 spectra will be acquired from 6.2 to $15.5 \mu \mathrm{m}$ and these data will be used in the analysis. Since variable path transmission will result from the varying nadir viewing angle, the data should be useful for water vapor transmission model verification, for testing the two-channel concept for sea surface temperature measurements, and for selection of a channel pair. In addition, the authors are currently working with personnel at Goddard Space Flight Center towards these objectives. The channel pair which is finally selected will be used on the Sea Surface Temperature Imaging Radiometer proposed for the Earth Observatory Satellite.

\section{References}

Bignell, K. J. (1970), Quan. J. R. Met. Soc. 96, 390-403. Roach, W. T. and R. M. Goody, (1958) Quant.J. R. Met. Soc. 84, 319-333.

Burch, D. D., Investigation of the Absorption of Infrared Radiation by Atmospheric Gases, Philco-Ford Corporation, Aeronutronic Division, Newport Beach, Calif. Report No. U-4784 (January 1970).

Altshuler, T. L., Infrared Transmission and Background Radiation by Clear Atmospheres, General Electric Company, Missile And Space Vehicle Department, Philadelphia, Penn., Document No. 61SD199 (December 1961). 